\title{
Acknowledgement to Reviewers of Environments in 2018
}

Environments Editorial Office

MDPI, St. Alban-Anlage 66, 4052 Basel, Switzerland

Published: 8 January 2019

Rigorous peer-review is the corner-stone of high-quality academic publishing. The editorial team greatly appreciates the reviewers who contributed their knowledge and expertise to the journal's editorial process over the past 12 months. In 2018, a total of 128 papers were published in the journal, with a median time to first decision of 19 days and a median time to publication of 43 days. The editors would like to express their sincere gratitude to the following reviewers for their cooperation and dedication in 2018:

$\begin{array}{lll}\text { Acharya, Tri Dev } & \text { Bareš, Vojtěch } & \text { Casse, Thorkil } \\ \text { Adamiec, Ewa } & \text { Batar, Amit } & \text { Castada, Hardy } \\ \text { Adams, James } & \text { Bayala, Jules } & \text { Castell, Nuria } \\ \text { Adeleye, Adeyemi } & \text { Beauchamp, Jonathan } & \text { Castellani, Beatrice } \\ \text { Afzal, Muhammad } & \text { Bekele, Dawit } & \text { Cavallo, Eugenio } \\ \text { Aivazidou, Eirini } & \text { Bermúdez, María } & \text { Ceddia, Michele Graziano } \\ \text { Akhtar, Fazlullah } & \text { Bian, Qijing } & \text { Cervero Aragó, Sílvia } \\ \text { Aletta, Francesco } & \text { Biasi, Rita } & \text { Chakrabarti Basu, } \\ \text { Alexander, Susan } & \text { Bičáková, Olga } & \text { Priyadarshini } \\ \text { Almeida, Ana Catarina } & \text { Biserni, Cesare } & \text { Chamberlain, James } \\ \text { An, Chunjiang } & \text { Biziuk, Marek } & \text { Chang, IPin } \\ \text { An, Jinwoo } & \text { Blaine, Tom } & \text { Chang, Yi-tang } \\ \text { Andersson, Morgan } & \text { Boguniewicz, Joanna } & \text { Charvat, Karel } \\ \text { Ando, Yoichi } & \text { Bohr, Joseph } & \text { Chaukura, Nhamo } \\ \text { Andrades-Peña, Francisco } & \text { Borge-Diez, David } & \text { Cheeseman, John } \\ \text { Javier } & \text { Borrego, Carlos } & \text { Chen, Liang-Yu } \\ \text { Andreoli, Enrico } & \text { Bozlaker, Ayse } & \text { Chen, Yaoning } \\ \text { Ariño, Cristina } & \text { Brandeis, Thomas } & \text { Chen, Yong } \\ \text { Arrebola, Francisco Javier } & \text { Brown, Kate Pride } & \text { Cheng, Chingwen } \\ \text { Aryal, Niroj } & \text { Bryant, Christopher } & \text { Chettiparamb, Angelique } \\ \text { Asci, Serhat } & \text { Burdon, Daryl } & \text { Chin, Cheng Siong } \\ \text { Asdrubali, Francesco } & \text { Butler, John } & \text { Choi, Juwon } \\ \text { Astolfi, Arianna } & \text { Caballero, Alvaro } & \text { Choi, Nag Jung } \\ \text { Aumond, Pierre } & \text { Caetano, Nídia } & \text { Choudhary, Kumari Sonal } \\ \text { Auvinen, Hannele } & \text { Cain, Daniel J. } & \text { Christopoulou, Anastasia } \\ \text { Ayuga Tellez, Francisco } & \text { Calabrò, Francesco } & \text { Chun, Jong Ahn } \\ \text { Azzone, Giovanni } & \text { Calabrò, Paolo S. } & \text { Coelho, J. Luis Bento } \\ \text { Bae, Hyokwan } & \text { Campagnolo, Manuel } & \text { Contini, Daniele } \\ \text { Bahmanteymouri, Elham } & \text { Campello-Vicente, Hector } & \text { Covino, Stefano } \\ \text { Bai, Shiqiang } & \text { Cao, Yuhe } & \text { Cresswell, Will } \\ \text { Baigorri, Artemio } & \text { Caravanos, Jack } & \text { Criss, Robert E. } \\ & & \\ & & \end{array}$


Crossman, Neville

Csurgai-Horváth, László

Cukrov, Neven

Cummings, Anthony

Czeszczewik, Dorota

Da Silva, Isabel Martinho

D'Alessandro, Francesco

Davies, Ian

Davis, Richard

de Freitas, Elisabete Fraga

De Jong, Wil

De La Rua Lope, Cristina

De Lourdes Gomes Pereira,

Maria

De Valck, Jeremy

Deb, Apurba Krishna

Delogu, Massimo

Denai, Mouloud

Dennington, Simon

Dennis, Matthew

Deryng, Delphine

Desrosiers, Melanie

Di Giuseppe, Dario

Dimitrova, Reneta

Dimova, Elitsa

Doroudiani, Saeed

Duarte, Lia

Duffy, Walter G.

Dzhonova, Daniela

Economou, Anastasios

Economou, Chris

Ejsmont, Jerzy

Elia, Mario

Elsaid, Khaled

Falasca, Serena

Falcone, Pasquale Marcello

Fan, Huan-Jung

Fan, Yuanchao

Farnood, Ramin R.

Feinn, Richard S.

Feng, Youcan

Fernandez, Begona

Fesenmaier, Daniel R.

Fialkowski, Wojciech

Field, Robert A.

Filchev, Lachezar H.

Fiore, Silvia

Foereid, Bente

Font, Josep
Fornaroli, Riccardo

Fredianelli, Luca

Friedrich, Theodor

Frontistis, Zacharias

$\mathrm{Fu}$, Xiao-An

Fuentes-Bargues, José Luis

Funge-Smith, Simon

Furman, Carrie

Gagliardi, Paolo

Galelli, Stefano

Gallo, Mariano

Gan, Luo

Ganser, Gary

Gato, Eric

Gharanjik, Ahmad

Giuffrida, Salvatore

Grabarczyk, Malgorzata

Grams, Heather M.

Granata, Francesco

Gutiérrez-Capitán, Manuel

Hackfort, Sarah K.

Han, Congzheng

Hardie, Mary

Hasanagas, Nikolaos D.

Haseneder, Roland

Haver, Darren L.

Hayakawa, Kazuichi

Healy, Eric W.

Hendel, Martin

Henkin, Zalmen

Henriksen, Hans Jørgen

Her, Younggu

Herckes, Pierre

Heutschi, Kurt

Hodge, Vernon

Hoffmann, Bernd

Hoghooghi, Nahal

Huang, Haiyan

Huang, Tianming

Huang, Xiao (Eric)

Huh, Taewook

Hurlbert, Margot A.

Hutchinson, Mark

Inaba, Atsushi

Iwasaki, Yuichi

Jackson, Scott

Jafarinejad, Shahryar

Janczukowicz, Wojciech

Jandl, Robert
Jantz, Patrick A.

Jaramillo, Fernando

Jeong, Kwangkook

Jerneck, Anne

Jin, Jianjun

Joffre, Olivier

Johansen, Chris

Johnson, Glenn

Joyce, Hannah

Julien, Pierre Y.

Jungbluth, Niels

Junge, Malte

Kaduk, Jörg

Kadyampakeni, Davie

Kahilainen, Kimmo K.

Kalamas Hedden, Maria

Kanakri, Shireen

Kandelbauer, Andreas

Karl, Matthias

Kasahara, Tamao

Kasak, Kuno

Kenny, Natasha

Khalid, Farrukh

Kidd, Kathryn

Kile, Molly

Killarney, James

Kim, Yun-Tae

Kimura, Nobuaki

Kirsanov, Dmitry

Klopp, Jacqueline M

Koeckler, Heike

Koehler, Manfred

Koehler, Tina

Kong, Xiangrui

Konstantakopoulou,

Foteini

Koo, Jawoo

Krishna Bahadur, KC

Król, Małgorzata

Krzywanski, Jaroslaw

Krzyżyńska, Renata

Kuo, Shu-lung

Kupriyanov, Alexander

Kurdve, Martin

Kusch-Brandt, Sigrid

Kwiecień, Iwona

Kyriakopoulos, Grigorios

L.

Kyriazopoulos, Apostolos 
Kyzas, George

Ladeira, Carina

Lamb, Dane T.

Law, David

Lee, Jaehyuck

Lee, Ming-Ching

Lee, Seungwoo

Leisher, Craig

Lekkas, Demetris Francis

Lerman, Zvi

Li, Ying

Li, Zijian

Lien, Gudbrand

Lin, Yuan-Chien

Liobikienè, Genovaitè

Lipscomb, Glenn

Liu, Chunhua

Liu, Lin

Liu, Wenping

Liu, Xi-chuan

Lo Giudice, Angelina

Lopez, Francesco

Louis, Jean-Nicolas

Loures, Luis

Lovarelli, Daniela

Macgregor, Nicholas

Magee, Darrin

Maggioni, Elena

Magrini, Anna

Mahbub, Parvez

Maksymiuk, Gabriela

Mallet, Cécile

Mallick, Bishawjit

Manana, Mario

Manzano-Agugliaro,

Francisco

Manzardo, Alessandro

Margaritis, Efstathios

Markondeya Raj,

Pulugurtha

Marques-Sanchez, Pilar

Martienssen, Marion

Martinelli, Luca

Martinez Del Castillo,

Edurne

Martinez, Antonio J Torija

Martínez-Vega, Javier

Mastrocicco, Micòl

Mateo-Lázaro, Jesús
Materia, Valentina

Cristiana

Matis, Kostas

McDonald, Walter

McDougall, Scott

McPhillips, Lauren

Milardović, Stjepan

Millar, Graeme

Miller, Glenn

Miller, Judith S.

Mochizuki, Junko

Moncada, Stefano

Monteiro, Cristina M.

Monteiro, Eliseu

Moore, Philip

Moorhouse, Heather

Morales-Marin, Luis

Moreno, Eduardo

Moser, Astrid

Mullaugh, Katherine

Muñoz Arnanz, Juan

Murillo Tovar, Mario

Alfonso

Musmarra, Dino

Nardi, Iole

Nastran, Mojca

Nebuloni, Roberto

$\mathrm{Ng}$, Bing Feng

Ngoy, Kikombo

Nicolini, Andrea

Nielsen, Gunnar D.

Nikolopoulos, Dimitrios

Norse, David

Northey, Stephen

Noussan, Michel

Noveron, Juan C.

Nuić, Ivona

Nunan, Fiona

Ognibene, Dimitri

Ojeda-Benitez, Sara

Okochi, Hiroshi

Oliveto, Giuseppe

Ongel, Aybike

Ortega, Marcelo F.

Osemwengie, Lantis

Österreicher, Doris

Ostoić, Silvija Krajter

Oyinlola, Muhammed A.

Ozalp, Nesrin
Pacheco-Torgal, Fernando

Pagella, Tim

Palella, Boris

Palella, Boris Igor

Palma, Luca Di

Palstra, S.W.L. (Sanne)

Pani, Shantanu Kumar

Panico, Antonio

Pant, Laxmi Prasad

Paolini, Riccardo

Pařílková, Jana

Pasanisi, Francesco

Pastorella, Fabio

Paulette, Laura

Pazdur, Anna

Pearce, Dora Claire

Pereira, Joana L.

Perez-Verdin, Gustavo

Perić, Mirela Sertić

Perry, Jim

Petrucci, Rita

Picchio, Rodolfo

Pirk, Christian

Pitarch, Elena

Pleasants, John M

Popp, József

Pourhashem, Ghasideh

Pufal, Gesine

Qiu, Guanglei

Race, Marco

Ramon, Peral Orts

Raszeja, Elżbieta

Razzaghmanesh, Mostafa

Reardon-Smith, Kathryn

Recanatesi, Fabio

Redon, Nathalie

Rega, Carlo

Rexha, Blerim

Rhi, Seok-ho

Rice, Lauren J.

Roche, Philip

Roggema, Rob

Rohde, Melissa M.

Roy, Sagar

Running, Katrina

Saafi, Mohamed

Saeidi, Nazanin

Safiuddin, Md.

Salata, Ferdinando 


Salazar, César
Sánchez Saavedra, María
del Pilar
Sánchez, Carles Flaquer
Sanchez, Miguel
Santiago-Freijanes, José
Javier
Santoro, Antonio
Sarkar, Sudipta
Saunders, Fred
Schelly, Chelsea
Schenone, Corrado
Schneibel, Anne
Schneider, Petra
Scholz, Bettina
Schröder, Lise
Schulte, Bruce
Scoccia, Rossano
Secondi, Luca
Semblante, Galilee
Seo, Youngmin
Shaffer, Gary
Shelia, Vakhtang
Shen, Ruiqing
Sherchan, Samendra
Shrestha, Mani
Shu, Hung-Yee
Sidorchuk, Aleksey
Siedenburg, Jules
Silibello, Camillo
Silva, Maria Barral
Skoczko, Iwona
Soltani, Salman
Sonetti, Giulia
Song, Jian
Spasiano, Danilo
Stafford, Gloria
Stenstrøm, Yngve
Sate

Straub, Ad D.

Sulzer, Philipp

Szabo, Judit

Taghizadeh-Mehrjardi,

Ruhollah

Tampakis, Stilianos

Tandy, Susan

Tangney, Peter

Taylor, Keith E

Teodoro, Ana Cláudia

Terbeck, Sylvia

Teschl, Franz

Thompson, Gregory J.

Thornhill, Ian

Tian, Nana

Tizaoui, Chedly

Todeschini, Sara

Tölle, Merja

Tong, Huanhuan

Touliatos, Dionysios

(Dennis)

Trachte, Katja

Tsaboula, Aggeliki

Tsagarakis, Konstantinos

Tsai, Kang-Ting

Uprety, Yadav

Urban, Noel

Urien, Nastassia

Urquijo Torres, Pedro

Sergio

Vagiona, Dimitra

Valipoor, Shabboo

Van Der Kwast, Johannes

Vasques, Gustavo

Vessio, Gennaro

Vicente, Gemma

Vidal, Tânia

Vílchez-Gómez, Rosendo
Waclawek, Stanislaw

Walmsley, Alena

Wang, Kuo-Lung

Wang, Shifeng

Wang, Ya-Fen

Ward, John

Wason, Jay

Waterlot, Christophe

Watkins, Bradley

Whitfield, Stephen

Wicks, Carol

Wilkie, Jim

Williams, Jason

Wilts, Hennning

Wink, Brian

Woolridge, Anne Christine

Wosniok, Werner

$\mathrm{Wu}$, Bing

$\mathrm{Wu}$, Songlin

$\mathrm{Wu}$, Zhentao

$X i e$, Hua

Yadav, Vikramaditya G.

Yakubu, Momoh

Yee, Susan

Yepes, Víctor

Yettella, Vineel

Younus, $\mathrm{Md}$

$\mathrm{Yu}$, Ting-To

Yustres, Ángel

Zambon, Giovanni

Zander, Kerstin K.

Zhang, Wei

Zhang, Wen

Zhao, Kaiguang

Zorrilla-Miras, Pedro

Źróbek-Sokolnik, Anna

Zwoździak, Anna

(C) 2019 by the authors. Licensee MDPI, Basel, Switzerland. This article is an open access article distributed under the terms and conditions of the Creative Commons Attribution (CC BY) license (http://creativecommons.org/licenses/by/4.0/). 